\title{
Computer-Generated van der Waerden Partitions
}

\author{
By R. S. Stevens and R. Shantaram
}

\begin{abstract}
An exhaustive search made on a PDP- 8 mini-computer shows the value of the van der Waerden number $n(2,5)$ to be 178 .
\end{abstract}

Let $S_{n}$ denote the set of natural numbers less than or equal to $n$. Van der Waerden's Theorem [2] may be stated as follows: For given natural numbers $k$ and $l$, there exists a natural number $n=n(k, l)$ such that for any $f: S_{n} \rightarrow S_{k}$, there is an arithmetic progression of length $l$ of arguments in $S_{n}$ on which $f$ is constant. In this note we compute the least positive $n=n(k, l)$ for some values of $k$ and $l$.

The proof given in [2] has much overkill and gives an upper bound for $n(k, l)$ that is theoretically constructive but for all practical purposes only existential.

For given $i, k$, and $l$, we define $f: S_{i} \rightarrow S_{k}$ to be valid if there is no arithmetic progression of the elements in $S_{i}$ of length $l$ on which $f$ is constant. Consider the following algorithm (for fixed $k$ and $l$ ) which is designed to find the largest value of $i$ for which $f: S_{i} \rightarrow S_{k}$ is valid. (Thus after termination, $i \max +1$ gives the smallest value of $n(k, l))$ :

STEP 1. Set $i=1, f(1)=1, i \max =1$.

STEP 2. Increment $i$ by 1 and then set $f(i)=1$.

STEP 3. If $f: S_{i} \rightarrow S_{k}$ is valid, then go to STEP 2 .

STEP 4. If $f(i)=k$, then go to STEP 6.

STEP 5. Increment $f(i)$ by 1 and then go to STEP 3.

STEP 6. If $i-1>i \max$, then set $i \max =i-1$.

STEP 7. Decrement $i$ by 1 .

STEP 8 . If $i>1$, then go to STEP 4 .

STEP 9. Stop.

This algorithm was programmed on a PDP-8 mini-computer and run with $k=2$ and various values of $l$. The resulting values of $i \max$ at termination and approximate running times are shown in the following table:

\begin{tabular}{cccc}
\hline$l$ & 3 & 4 & 5 \\
\hline$i \max$ & 8 & 34 & 177 \\
time & instantaneous & 3 seconds & 210 hours \\
\hline
\end{tabular}

What is perhaps somewhat more interesting is the nature of the valid functions $f: S_{i \max } \rightarrow S_{2}$. For purposes of illustration we use the two symbols 0 and 1 (rather than 1 and 2 ) and indicate the functions merely by strings of 0 's and 1's. We 
indicate only the essentially different strings that were found. All others can be obtained by relabeling (i.e., by interchanging the 0's and 1's).

In the case $l=3$, there are three strings: 00110011, 01011010, and 01100110 .

In the case $l=4$, there is a repeating subsequence of length 10 , which we denote by $X=0100011101$. Then all strings are of the form ${ }^{*} X^{*} X^{*} X^{*}$, where the *'s (called freebies) denote single entries whose only restriction is that there be no arithmetic string of four freebies all alike. There are 14 valid strings of length 34 .

In the case $l=5$, the repeating subsequence structure is "two deep." More precisely, let $X=0010000100$ and $Y=0111010001$. Let $X^{\prime}$ and $Y^{\prime}$ be obtained from $X$ and $Y$, respectively, by relabeling. Then the subsequence $Z$ is $X^{*} Y^{*} X^{*} Y^{\prime}$ (as before, the *'s denote freebies). The valid strings of length 177 are all of the form ${ }^{*} Z^{*} Z^{*} Z^{*} Z^{*}$ or reversed from this (end for end). Note that by virtue of the symmetry exhibited by $Z$, we may cyclically "conjugate" and obtain, for example, a reversal by instead setting $Z=Y^{*} X^{*} Y^{*} X$. These conclusions about the subsequence structure were arrived at in the following way:

The initial string 00010000100 was given (i.e., first freebie set to 0 and next 10 symbols set to $X$ ) to the computer. Sufficient output was printed to establish that $Z$ must be constructed and repeated as given above in order to obtain a valid string of length 177 . Then, noting that there are 17 freebies, a count was made of all valid strings of length 17 with first symbol 0, yielding 24,203. This compares exactly with a count made of all valid strings of length 177 with initial string 00010000100 .

A check via computer output also showed that the first 11 symbols must be initialized to *U ( $U$ is one of the four "blocks" $X, Y, X^{\prime}$, or $Y^{\prime}$ shown above), and that the appropriate "conjugate" of $Z$ must be constructed and repeated in order to obtain valid strings of length 177 .

In conclusion, we note that $n(2,5)=178$ found above extends the table on page 31 prepared by V. Chvatal [1]. We would also like to record our agreement with the value $n(3,3)=27$ found in this reference.

Department of Mathematics

The University of Michigan-Flint

Flint, Michigan 48503

1. V. CHVATAL, Some Unknown van der Waerden Numbers, Symposium in Combinatorial Methods, Calgary, Alberta, Canada, 1969, pp. 31-33.

2. A. Y. KHINCHIN, Three Pearls of Number Theory, Graylock Press, Rochester, New York, 1952. 